Article

\title{
Evaluation of the Effect of Operating Parameters on Thermal Performance of an Integrated Starter Generator in Hybrid Electric Vehicles
}

\section{Moo-Yeon Lee ${ }^{1}$, Dong Hyun Lim $^{2}$ and Sung Chul Kim ${ }^{3, *}$}

1 Department of Mechanical Engineering, Dong-A University, 37 Nakdong-Daero 550, Saha-gu, Busan 604-714, Korea; E-Mail: mylee@dau.ac.kr

2 Green Car Power System R\&D Division, Korea Automotive Technology Institute, 74 Yongjung-Ri, Pungse-Myun, Dongnam-Gu, Chonan-Si, Chungnam 330-912, Korea; E-Mail: dhlim@katech.re.kr

3 School of Mechanical Engineering, Yeungnam University, 280 Daehak-Ro, Gyeongsan, Gyeongbuk 712-749, Korea

* Author to whom correspondence should be addressed; E-Mail: sungkim@ynu.ac.kr; Tel.: +82-53-810-2572; Fax: +82-53-810-4627.

Academic Editor: Joeri Van Mierlo

Received: 6 March 2015 / Accepted: 20 August 2015 / Published: 24 August 2015

\begin{abstract}
The belt-driven-type integrated starter generator motor in a hybrid electric vehicle is vulnerable to thermal problems owing to its high output power and proximity to the engine. These problems may cause demagnetization and insulation breakdown, reducing the performance and durability of the motor. Hence, it is necessary to evaluate the thermal performance and enhance the cooling capacity of the belt-driven type Integrated Starter Generator. In this study, the internal temperature variations of the motor were investigated with respect to the operating parameters, particularly the rotation speed and environment temperature. At a maximum ambient temperature of $105^{\circ} \mathrm{C}$ and rotation speed (motor design point) of $4500 \mathrm{rpm}$, the coil of the motor was heated to approximately $189^{\circ} \mathrm{C}$ in generating mode. The harsh conditions of the starting mode were analyzed by assuming that the motor operates during the start-up time at a maximum ambient temperature of $105^{\circ} \mathrm{C}$ and rotation speed (motor design point) of $800 \mathrm{rpm}$; the coil was heated to approximately $200{ }^{\circ} \mathrm{C}$, which is close to the insulation temperature limit. The model for analyzing the thermal performance of the ISG was verified by comparing its results with those obtained through a generating-mode-based experiment.
\end{abstract}


Keywords: thermal performance; integrated starter generator; motor; operating parameter; hybrid electric vehicle

\section{Introduction}

The demand for hybrid vehicles is increasing along with the interest shown by consumers for environmentally friendly automobiles. A hybrid vehicle consists of a motor for driving as well as various electric and electronic systems aimed to improve the fuel efficiency and performance. The power consumption of a vehicle increases with the addition of devices for driver safety or convenience. Hence, the required power capacity of the vehicle-mounted generator also increases; this has created the need for efficient power generation to ensure stable operation of application components and to improve the fuel efficiency of the vehicle [1-3].

In hybrid vehicles, an Integrated Starter Generator (ISG) is a component that regulates both the starter motor and generator. The ISG can stably satisfy increases in the power demand and it is a key component for realizing additional fuel efficiency improvements owing to its idle stop and go function (to reduce fuel consumption when idling) and its potential for regenerative breaking (to recover the vehicle's inertial energy as electric energy when the vehicle decelerates) [4,5]. Unlike the direct-coupled-type motor, which is directly coupled to the engine's crankshaft, the belt-operated ISG can be applied without significant alterations to the engine design because it employs connection methods similar to those of existing vehicle-mounted generators. It is installed near the $1.2-1.5 \mathrm{~kW}$ vehicle-mounted alternator and it requires a high power density to meet the output level of $5 \mathrm{~kW}$.

A high power density increases the motor loss density, which can increase the temperature in the motor. This may cause demagnetization of the permanent magnet and insulation breakdown of the coil conductor, reducing the performance and durability of the motor and casing mechanical failure in the machine [6-9]. Motors with a high power density generally employ water- or oil-based cooling methods that use a coolant or oil with a high thermal capacity; however, such methods require a separate space for the cooling channel inside the motor and are inconvenient because they require a connection with an exterior heat exchanger. Forced-air-cooling is advantageous since it has a simple cooling structure design; however, its radiation characteristics may easily change with the driving conditions or surrounding environment. As shown in Figure 1, the ISG connected to the engine crankshaft is directly affected by the engine heat, and its thermal performance can easily be changed by the fan when it operates as a starter or generator under various conditions. Hence, ISG not only have to satisfy high efficiency requirements but they would also require a radiation design that can effectively remove the heat generated by motor loss. 


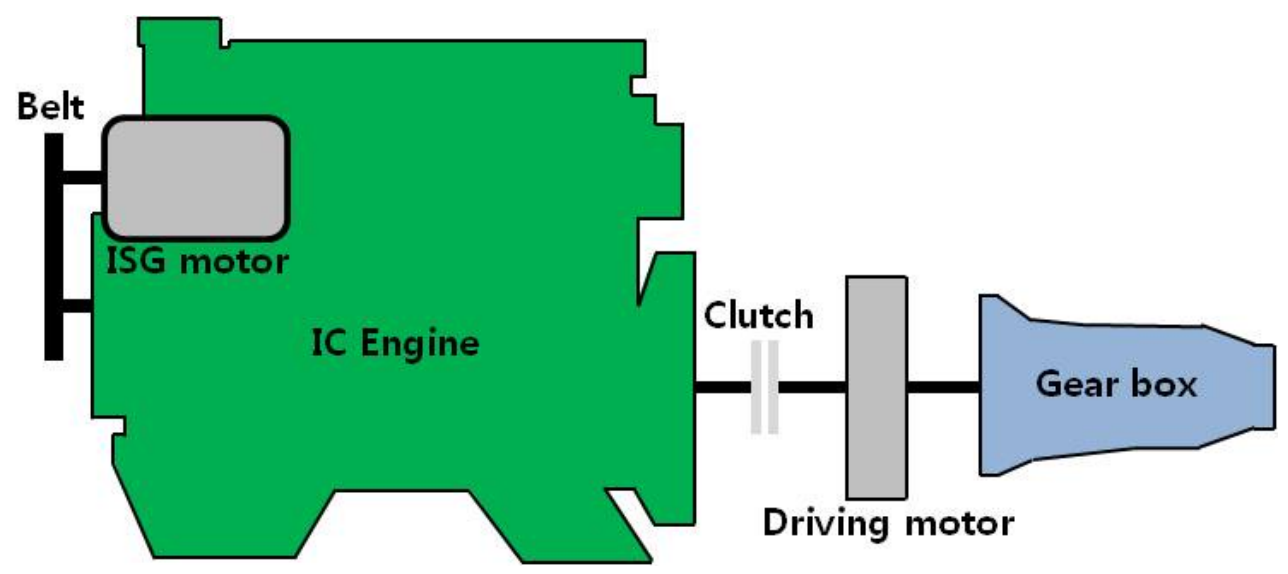

Figure 1. Schematic diagram of the Integrated Starter Generator (ISG) system for HEVs (hybrid electric vehicles) [4].

Various studies on topics ranging from motor performance to thermal flow interpretation and thermal performance improvements have been conducted for the development of motors. Kimotho, et al. [10] used computational fluid dynamics (CFD) analysis to observe the effect of the form of a housing pin on the thermal performance of a brushless direct current (BLDC) permanent magnet motor used in electric vehicles. They showed that CFD analysis can be used to optimize the thermal performance of high-capacity electric vehicle motors and improve the thermal performance by a maximum of $15 \%$ based on the end winding temperature. Lim, et al. [11] performed experiments on radiation and analyzed the heat flow of an oil spray cooling-type in-wheel motor and assessed the temperature and heat transfer characteristics of each major component with respect to the driving conditions. Kim, et al. [12] analyzed the thermal flow of a water-cooled ISG using a thermal equivalent circuit network. Their analysis results confirmed there was an error of approximately 10\% compared to the experimental results, and they showed that it was possible to perform a temperature analysis and predict the thermal properties of a motor by using an equivalent thermal circuit network during the preliminary design.

The present study analyzes the effects of the operating parameters through a thermal flow analysis considering the ambient temperature and rotation speed under generator-mode-operating conditions of a prototype $42 \mathrm{~V}$ ISG. A transient thermal flow analysis was conducted under driving conditions to analyze changes in the short-term temperature increase and heat dissipation characteristics. The performance of the motor dynamometer was compared with the thermal flow analysis results to verify the applicability of the heat value and analysis model used in the thermal flow analysis.

\section{Numerical Analysis and Experimental Setup}

\subsection{Motor Design and Performance Characteristics}

In this experimental study, the belt-drive-type ISG was designed to deliver a maximum torque of $150 \mathrm{Nm}$ to the axis for a belt rate of $2.5: 1$, generating $60 \mathrm{Nm}$ at $800-1500 \mathrm{rpm}$ to operate the vehicle. A wide operating range of 3000-15,000 rpm was considered for the generation section of the ISG. The design specifications were set to a maximum output of $5.7 \mathrm{~kW}$ and over $90 \%$ efficiency at $4500 \mathrm{rpm}$, which was the rotational speed range for driving the vehicle at a constant speed. The performance of the ISG was characterized with a finite element model (FEM) analysis program [13]. 
As a starter, the designed ISG can output a starting torque of $60 \mathrm{Nm}$ and power of $5 \mathrm{~kW}$ at $80 \%$ efficiency at 800-1500 rpm. A maximum output of $5.7 \mathrm{~kW}$ at $92 \%$ efficiency was recorded when the load was $12 \mathrm{Nm}$ at $4500 \mathrm{rpm}$; thus, the design objectives were satisfied. Additional design objectives were satisfied when a $5 \mathrm{~kW}$ output at $89 \%$ efficiency was recorded for the maximum speed of $15,000 \mathrm{rpm}$. Table 1 lists the specifications required to realize the above characteristics.

Table 1. Specifications of ISG.

\begin{tabular}{cc}
\hline Components & Value \\
\hline Maximum output $(\mathrm{kW})$ & 5.7 \\
\hline Maximum speed $(\mathrm{rpm})$ & 15,000 \\
\hline Rated current $(\mathrm{A})$ & 150 \\
\hline Elec. time constant $(\mathrm{ms})$ & 22.06 \\
\hline Mech. time constant $(\mathrm{ms})$ & 31.62 \\
\hline Maximum current $(\mathrm{A})$ & 330 \\
\hline Back-EMF constant per phase $(\mathrm{V})$ & 16.59 \\
\hline Efficiency $(\%)$ & 92 \\
\hline Phase resistance $(\Omega)$ & 0.0071 \\
\hline Phase inductance & 0.3569 \\
\hline
\end{tabular}

The temperature varied significantly with respect to the environmental conditions in the vehicle engine room and ISG loss. Because the temperature variation affects the durability and may cause malfunctions in the motor, cooling and performance designs were employed. The housing-less ISG type employs an air-cooling method using centrifugal cooling fans installed on both sides of the motor, as shown in Figure 2. The motor part typically consists of a rotor and stator. The rotor comprised a rotor core, permanent magnet $(\mathrm{NdFeB})$, shaft, etc., and the stator included a stator core and coil $(\phi 0.75)$. The temperature limit of the coil insulation is about $200{ }^{\circ} \mathrm{C}$. A flange and bracket were installed on both sides of the motor to protect components such as the cooling fans, bearings, and resolvers. The flange and bracket were locked with bolts to fix the ISG when it is installed in the vehicle. The motor employed heat protection designs with improvements verified through thermal flow interpretation and analysis of the initial and improved ISG, as reported by Kim, et al. [14].

Improving the shape of the cooling fan blade and the changing of locations of the entry and exit holes of the flange and bracket installed on the ISG significantly decreased the flow recirculation and improved the thermal performance. The flow resistance was reduced when the number and angle of the exit holes of the flange and bracket were adjusted. This in turn increased the air volume, which improved the thermal performance. 


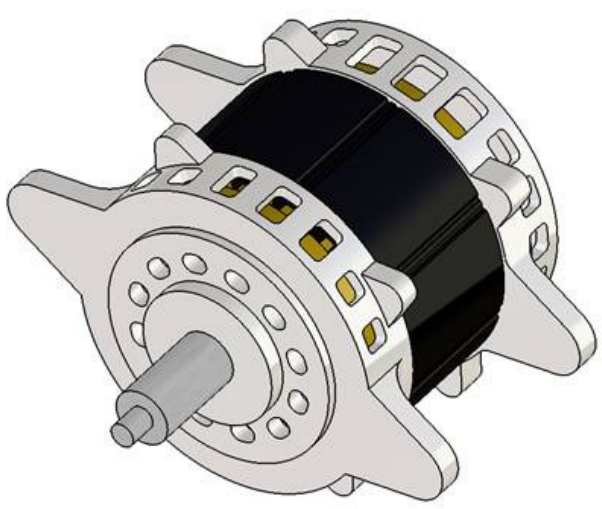

(a)

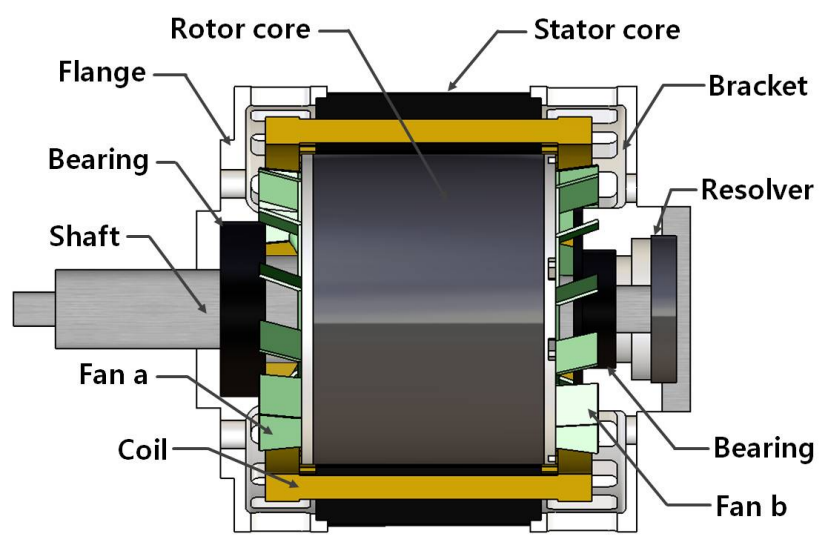

(b)

Figure 2. External and internal views of the ISG. (a) External view. (b) Internal view.

\subsection{Analysis Model and Boundary Condition}

Thermal flow analysis of the ISG was conducted using commercial CFD software [15]. The mass, momentum, and energy conservation equations were used as the governing equations for the simulation of the ISG. The turbulence model is used for calculating a turbulent flow using constants obtained via empirical and theoretical methods. The modified production (MP) k- $\varepsilon$ equation, which is known as the Kato-Laundar modification, was used for the turbulence model [16]. In the MP k- $\varepsilon$ turbulence model, the formation term of the standard k- $\varepsilon$ equations is adjusted to compensate for overestimation of the formation near the stagnation point, which appears in the standard k- $\varepsilon$ equations.

The shapes for interpretation in the thermal analysis model were either adjusted or simplified. This increased the accuracy of the analysis of the flow region around the surface of the solid; the model used for the analysis was made similar to the actual form by using an analysis mesh created by combining tetrahedron and prism elements. Approximately 14 million mesh elements were used for the analysis, as shown in Figure 3.

Because the ISG is located close to the engine, it may be exposed to extreme environments, with a maximum temperature of $105{ }^{\circ} \mathrm{C}$ under extreme summer conditions. Hence, a room temperature of $25^{\circ} \mathrm{C}$ and extreme temperature of $105^{\circ} \mathrm{C}$ were considered to be the environmental conditions, and a thermal flow analysis was carried out in $20^{\circ} \mathrm{C}$ increments. The pulley speed ratio of the ISG was assumed to be $2.5: 1$; the heat protection characteristics of the major components were analyzed based on the ISG operating conditions, in increments of $1500 \mathrm{rpm}$ in the generator speed range from $3000 \mathrm{rpm}$ to $15,000 \mathrm{rpm}$. The transient state thermal flow was analyzed to predict the component temperature, which changes abruptly within a short period owing to high loss in the startup range of the ISG. The moving mesh method was used to simulate the motor rotation. The motor rotating at $800 \mathrm{rpm}$ for $10 \mathrm{~s}$ was analyzed in time steps of $0.0024 \mathrm{~s} /$ cycle for approximately 2.5 days. 


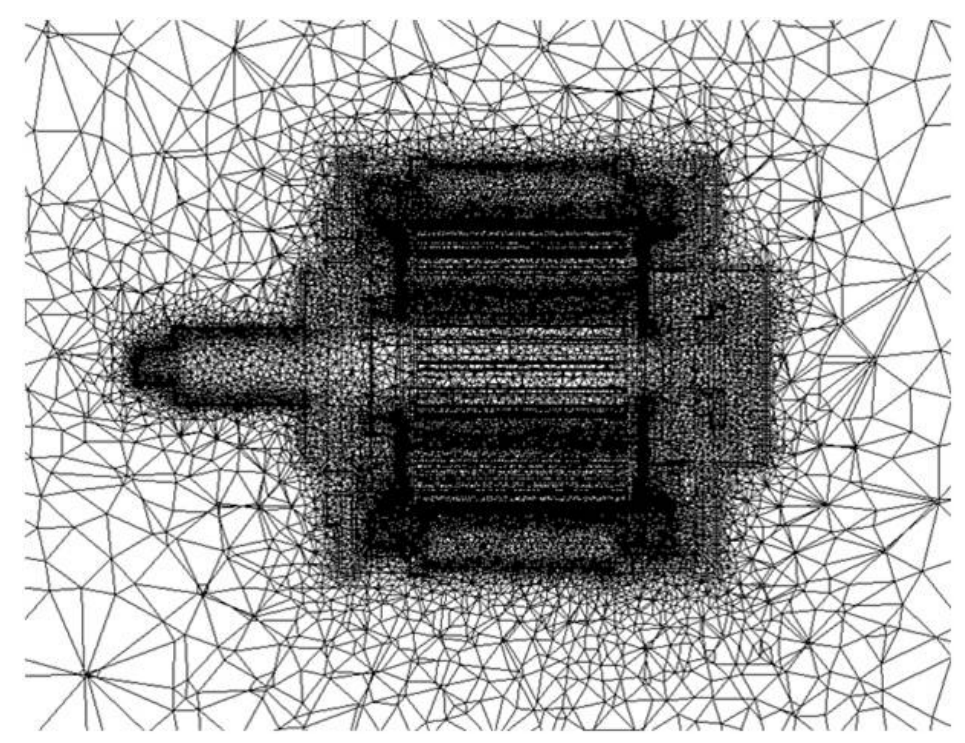

Figure 3. Grid model of the entire configuration.

In the analysis, the air around the ISG was assumed to be a three-dimensional (3D) normal incompressible turbulent flow. The material properties of each component in the analysis were set to be constant regardless of the temperature, as indicated in Table 2.

Table 2. Material properties of ISG parts.

\begin{tabular}{ccccc}
\hline Part name & Material & $\begin{array}{c}\text { Density } \\
\left(\mathbf{k g} / \mathbf{m}^{\mathbf{3}}\right)\end{array}$ & $\begin{array}{c}\text { Specific heat } \\
\left(\mathbf{J} / \mathbf{k g} \cdot{ }^{\circ} \mathbf{C}\right)\end{array}$ & $\begin{array}{c}\text { Thermal conductivity } \\
(\mathbf{W} / \mathbf{m} \cdot \mathbf{K})\end{array}$ \\
\hline Flange & $\mathrm{A} 6061$ & 2698 & 896 & 180 \\
\hline Bracket & $\mathrm{A} 6061$ & 2698 & 896 & 180 \\
\hline Stator core & $\mathrm{S} 18$ & 7650 & 486 & 50.8 \\
\hline Rotor core & $\mathrm{S} 18$ & 7650 & 486 & 50.8 \\
\hline Shaft & $\mathrm{SM} 54 \mathrm{C}$ & 7650 & 486 & 50.8 \\
\hline Coil & $\mathrm{Cu}$ & 8660 & 385 & 128 \\
\hline Magnet & $\mathrm{NdFeB}$ & 7500 & 460 & 9 \\
\hline Bearing & $\mathrm{STS} 420 \mathrm{~J} 2$ & 7900 & 460 & 50.9 \\
\hline Fan & $\mathrm{SM} 45 \mathrm{C}$ & 7650 & 486 & 50.8 \\
\hline Resolver & $\mathrm{S} 18$ & 7650 & 486 & 16.2 \\
\hline Magnet support & $\mathrm{STS} 304$ & 8000 & 500 &
\end{tabular}

In the motor, the representative causes of heat are the mechanical loss of the bearing, iron loss generated in the core, and copper loss generated in the coil. The copper loss was determined from the current flowing in the coil and internal resistance, as expressed by Equation (1). The resistance of the three-phase coil in Equation (1) can be calculated via Equation (2) [17]. The iron loss generated by the eddy current and hysteresis losses can be expressed by Equation (3) and can be obtained by considering the material of the core and the peak magnetic flux density $B . k h, k e$, and $n$ denote the hysteresis constant, eddy current constant, and Steinmetz constant, respectively [18]. The mechanical loss generated in a motor rotating at a high speed and representative losses such as copper loss and iron loss are generated by friction loss and wind loss, as shown in Equation (4). Friction loss is generated by the friction 
resistance of the bearing, as shown in Equation (5), and was considered in the present study. However, the wind loss, which is relatively small, was ignored [19].

Copper loss

$$
\begin{gathered}
P_{\text {copper }}=3 \times R_{p h} \times I_{\text {eff }}^{2}=3 R_{p h} \times\left(\frac{i_{\text {max }}}{\sqrt{2}}\right)^{2} \\
R_{p h}=R_{c u}\left(T_{c u}\right) \times \frac{n_{s p} L_{s p}}{S_{c}}
\end{gathered}
$$

Iron loss

$$
P_{\text {iron }}=P_{h}+P_{e}+P_{a}=k_{h} f B^{n}+k_{e} f^{2} B^{2}+k_{a} f^{1.5} B^{1.5}
$$

Mechanical loss

$$
\begin{gathered}
P_{\text {mech }}=P_{\text {fric }}+P_{\text {wind }} \\
P_{\text {mech }} \approx P_{\text {fric }}=\frac{3}{2} \times n_{r} \times m_{\text {rot }} \times N^{3} \times 10^{-6}
\end{gathered}
$$

The adjusted heat value, which reflects the effect of changes in the external environment temperature and rotation on each analysis condition, was applied. Tables 3 and 4 list the boundary conditions and thermal capacity applied in the thermal flow analysis of the ISG, respectively. The moving wall condition was applied to the simple cylindrical rotor core and shaft to simulate the operation of the motor's rotator. The multiple reference frame (MRF) method, which applies a gyro effect by creating a virtual rotation volume though the separation of the flow region around the rotor into rotational and non-rotational parts, was applied to the centrifugal cooling fan. To reflect the degree of convergence of the dependent variables during iterations, the variables were considered to have converged when the maximum relative error was $10^{-6}$ or less for the continuity, momentum, turbulence, and energy equations.

Table 3. Boundary conditions.

\begin{tabular}{cc}
\hline Components & Conditions \\
\hline Ambient temp. $\left({ }^{\circ} \mathrm{C}\right)$ & $25 *, 45,65,85,105$ \\
\hline Ambient pressure $(\mathrm{Pa})$ & Atmospheric pressure \\
\hline Motor speed $(\mathrm{rpm})$ & $800,3,000,4,500 *, 6,000,7,500,9,000,10,500,12,000,13,500,15,000$ \\
\hline
\end{tabular}

* Standard condition 
Table 4. Heat source variations with respect to the design and operating parameters.

\begin{tabular}{|c|c|c|c|c|c|c|}
\hline \multicolumn{3}{|c|}{ Operating Condition } & \multicolumn{3}{|c|}{ Part Loss (W) } & \multirow{2}{*}{ Total Loss (W) } \\
\hline Speed (rpm) & Torque $(\mathbf{N} \cdot \mathbf{m})$ & Temp. $\left({ }^{\circ} \mathrm{C}\right)$ & Coil & Stator & Bearing & \\
\hline 800 & 59.64 & 105 & 2360.8 & 14.5 & 1.79 & 2377.1 \\
\hline 3,000 & 18.56 & 25 & 459.5 & 20.4 & 13.0 & 492.9 \\
\hline \multirow{5}{*}{4,500} & \multirow{5}{*}{13.33} & 25 & 459.5 & 17.9 & 23.9 & 501.3 \\
\hline & & 45 & 494.9 & 17.8 & 23.9 & 536.6 \\
\hline & & 65 & 530.3 & 17.7 & 23.9 & 571.9 \\
\hline & & 85 & 565.8 & 17.7 & 23.9 & 607.4 \\
\hline & & 105 & 601.2 & 17.6 & 23.9 & 642.7 \\
\hline 6,000 & 10.27 & \multirow{7}{*}{25} & 459.5 & 18.4 & 36.9 & 514.8 \\
\hline 7,500 & 8.47 & & 459.5 & 20.2 & 51.5 & 531.2 \\
\hline 9,000 & 7.03 & & 459.5 & 22.4 & 67.7 & 549.6 \\
\hline 10,500 & 6.13 & & 459.5 & 25.5 & 85.3 & 570.3 \\
\hline 12,000 & 5.77 & & 459.5 & 29.5 & 104.3 & 593.3 \\
\hline 13,500 & 4.86 & & 459.5 & 33.0 & 124.4 & 616.9 \\
\hline 15,000 & 4.32 & & 459.5 & 37.9 & 145.7 & 643.1 \\
\hline
\end{tabular}

\subsection{Experimental Setup and Method}

The performance of the ISG prototype was evaluated to validate the reliability of the thermal flow analysis results. Figure 4 shows the ISG prototype used in the evaluation. The performance data and steady-state temperature at each major location with respect to the operating parameters were collected.

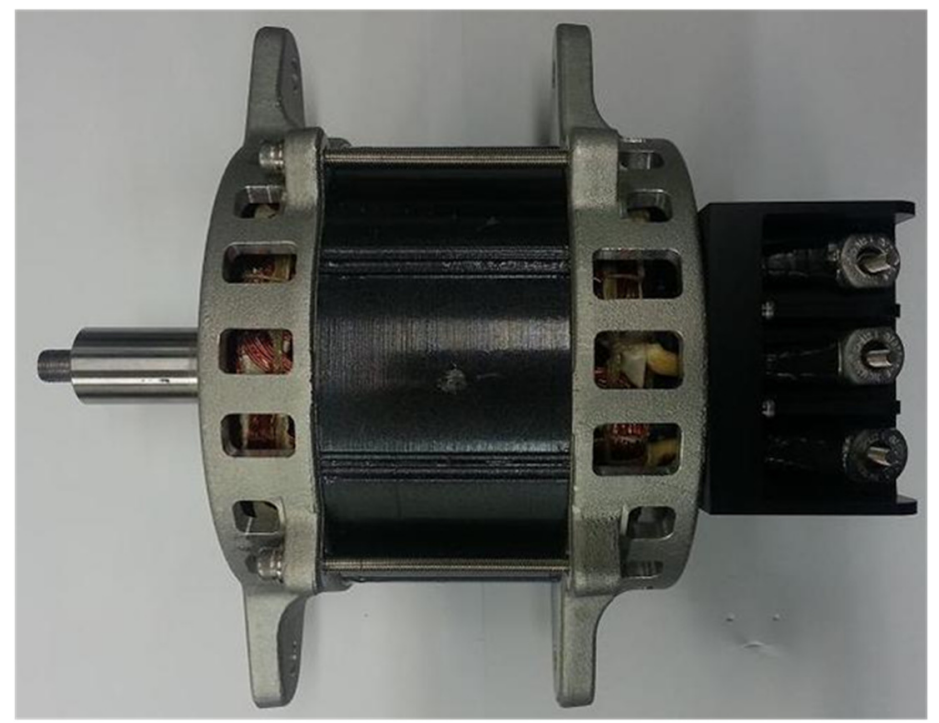

Figure 4. Prototype of the ISG.

Figure 5 shows the various pieces of apparatus used to measure the motor performance and steady-state temperature of each major component. The apparatus comprised a motor dynamometer with a mounting jig, controller, and measurement instrument. A T-type thermocouple with a measurement error of $\pm 0.1{ }^{\circ} \mathrm{C}$ collected the surface temperature data at the stator and at three points at the coil end. The temperature was considered to have reached saturation when its variation at each location was within 
$\pm 0.25{ }^{\circ} \mathrm{C}$ for $10 \mathrm{~min}$. The climatic chamber $(1000 \mathrm{~W} \mathrm{~mm} \times 1000 \mathrm{H} \mathrm{mm} \times 1000 \mathrm{D} \mathrm{mm})$ was used to simulate the external environmental condition of $25^{\circ} \mathrm{C}$. The environmental temperature can be modified in order to perform tests at different environmental temperature by controlling the heater and refrigeration system.

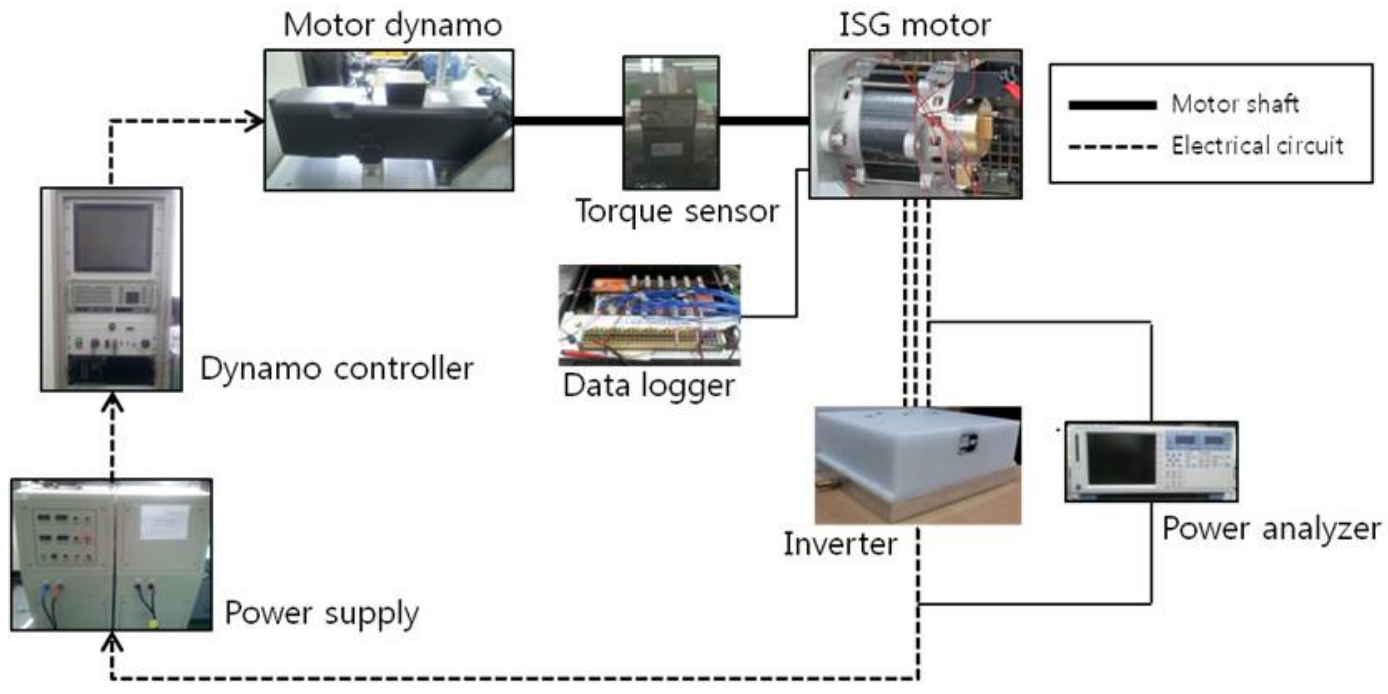

Figure 5. Experimental apparatus to measure motor performance and steady state temperature of the ISG components.

Figure 6 shows the picture of the experimental apparatus to measure thermal performance. Table 5 lists the specifications of the ISG thermal performance test equipment. A load motor that could reach a maximum of $18,000 \mathrm{rpm}$ at $7.5 \mathrm{~kW}$ was used for the motor dynamometer. The torque transducer installed with the motor dynamometer took measurements up to $20 \mathrm{Nm} / 32,000 \mathrm{rpm}$ and had a $0.1 \%$ measurement error. The power analyzer used to measure the efficiency can measure voltages of $15-1000 \mathrm{~V}$ and currents of $500 \mathrm{~mA}-30$ A with a $0.02 \%$ error.

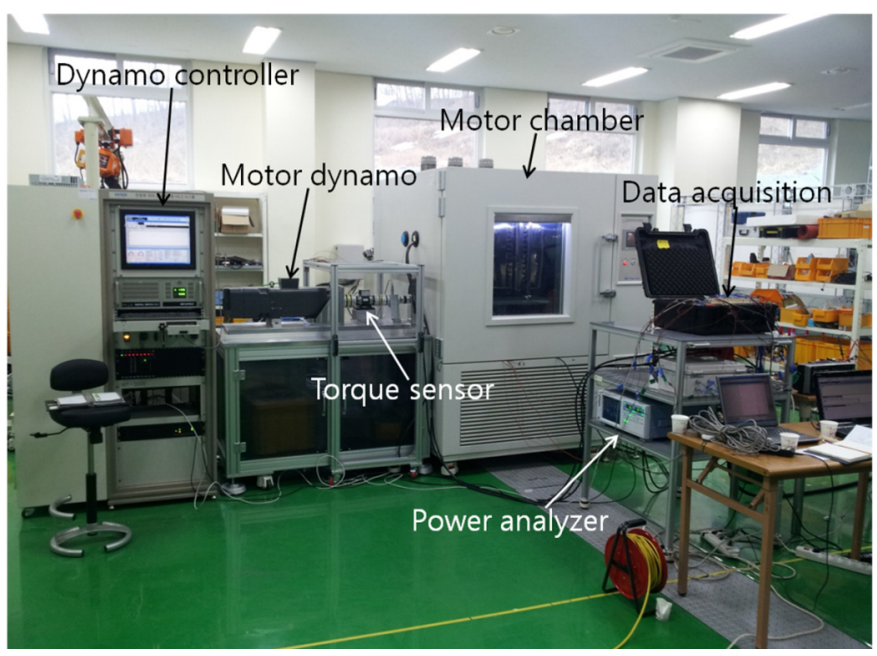

Figure 6. Picture of the experimental apparatus. 
Table 5. Specifications of test equipment.

\begin{tabular}{cc}
\hline Items & Specifications \\
\hline Thermocouples (T-type) & -200 to $400{ }^{\circ} \mathrm{C}\left( \pm 0.1{ }^{\circ} \mathrm{C}\right)$ \\
\hline Data logger (Gantner) & E. Gate IP $(\mathrm{V} 3)(2.93 \mathrm{~W} @ 12.06 \mathrm{~V})$ \\
\hline Motor dynamometer (Siemens) & $7.5 \mathrm{~kW} / 18,000 \mathrm{rpm} / 18 \mathrm{Nm}$ \\
\hline Torque transducer $(\mathrm{HBM})$ & $20 \mathrm{Nm}( \pm 0.1 \%)$ \\
\hline RPM sensor (HBM) & $32,000 \mathrm{rpm}( \pm 0.01 \%)$ \\
\hline Power analyzer (Yokogawa) & 15 to $1000 \mathrm{~V}( \pm 0.02 \%)$ \\
\hline
\end{tabular}

\section{Results and Discussion}

\subsection{Effects of Operating Parameters in Generating Mode}

Figure 7 shows the cross-section of the temperature distribution the ISG machine operating at $4500 \mathrm{rpm}$ at a room temperature of $25^{\circ} \mathrm{C}$. The coil and stator maintained high temperatures because the heat generation in the ISG was concentrated at these parts. A centrifugal cooling fan was installed to prevent malfunction caused by dielectric breakdown due to degradation resulting from the coil's prolonged exposure to high temperatures [8,9]; the fan sent the flow into the flange and bracket, as shown in Figure 8. The flow produced by the centrifugal cooling fan cooled the ISG as it exchanged heat with not only the coil and stator but also the flange and bracket.

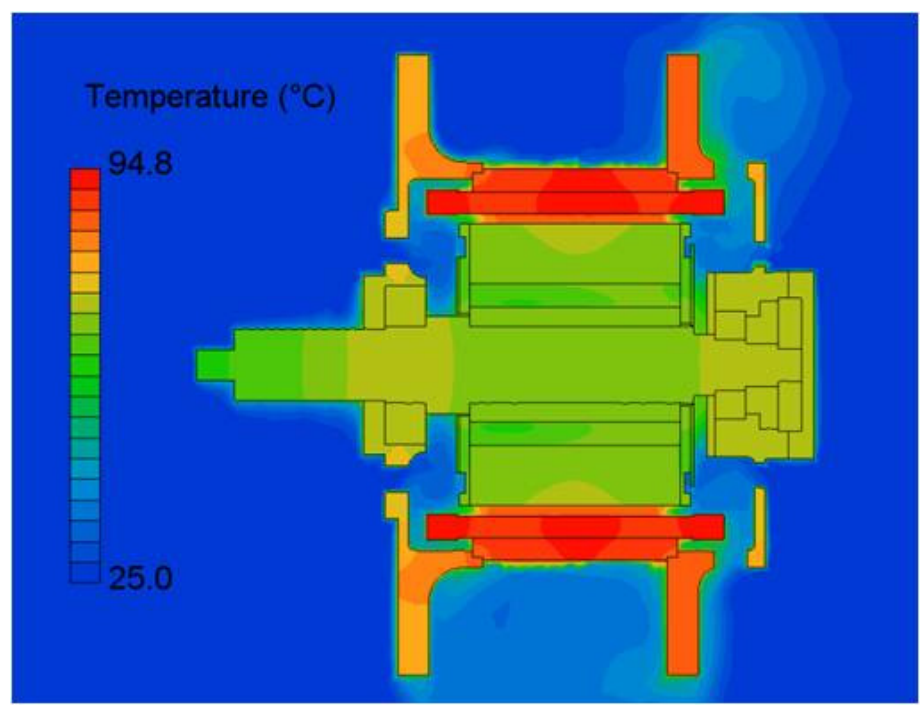

Figure 7. Temperature contours of the ISG operating at an ambient temperature of $25^{\circ} \mathrm{C}$ and a motor speed of $4500 \mathrm{rpm}$. 


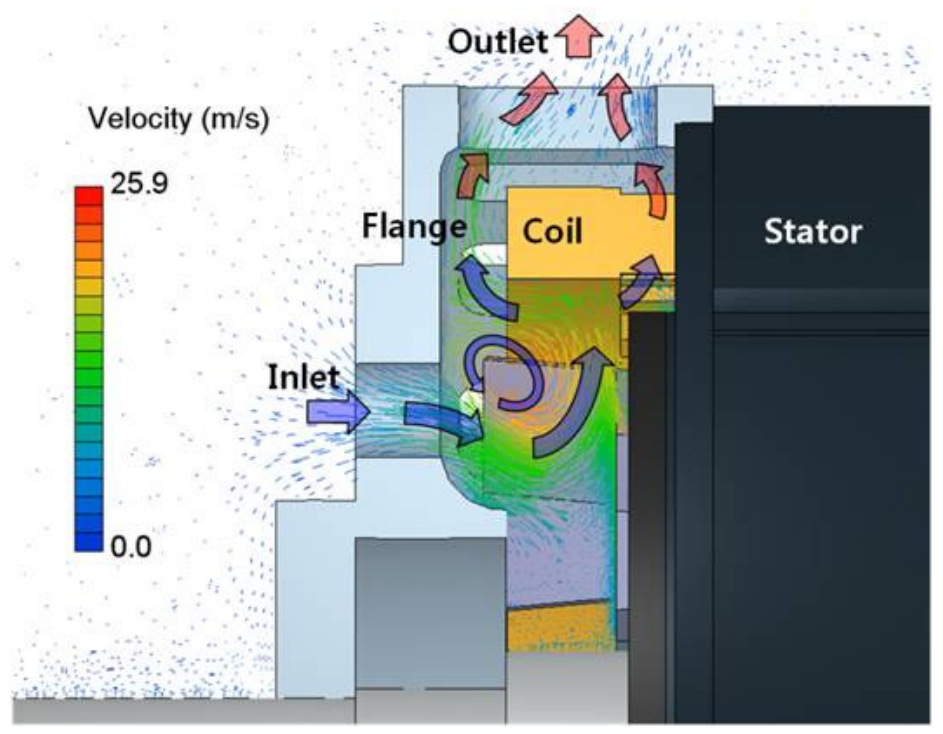

Figure 8. Internal flow of the ISG operating at an ambient temperature of $25^{\circ} \mathrm{C}$ and a motor speed of $4500 \mathrm{rpm}$.

Because the thermal and heat dissipation characteristics of an ISG employing the forced air-cooling method may change with the operating speed and environment temperature, a thermal flow analysis was conducted with varying operating parameters. The thermal flow analysis showed that the heat generated by copper, iron, and mechanical losses was discharged along the path shown in Figure 9 through conduction and convection (radiation effects were ignored). Some of the heat generated in the coil and stator of the forced air-cooled ISG was directly lost via the forced flow produced by the cooling fan's rotatory power. The rest of the heat was transferred through the contact surface of the coil, stator, flange, and bracket by conduction and then from the flange and bracket to the air by convection. Most of the heat generated at the bearing, on the other hand, was lost through conduction from the flange, bracket, and shaft.

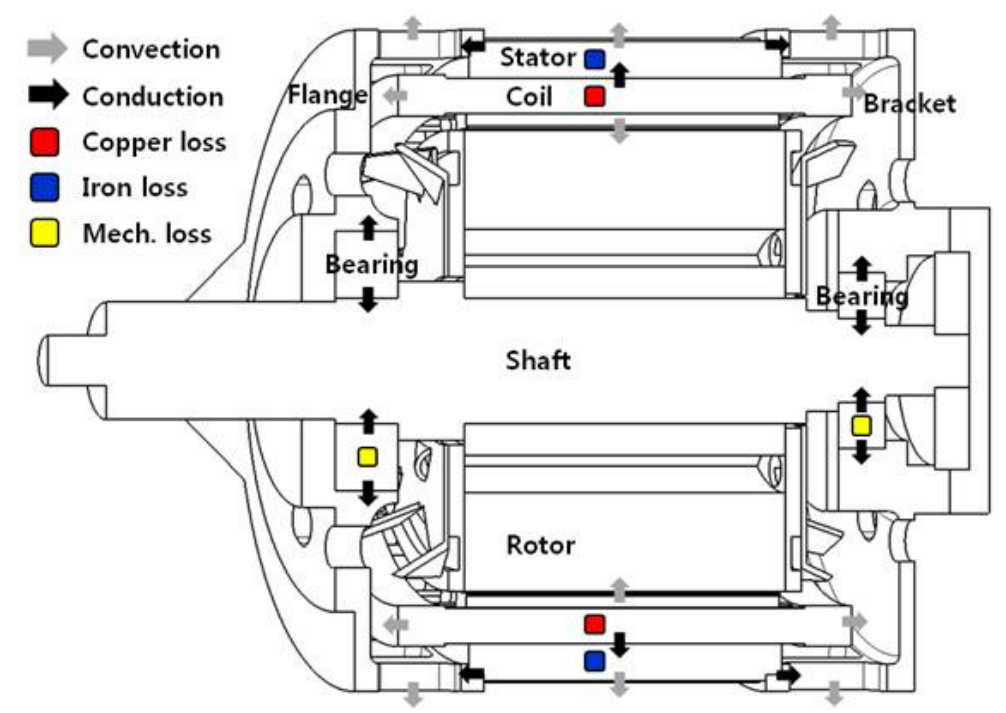

Figure 9. Heat transfer paths in the ISG. 
The changes in the heat dissipation characteristics with respect to the operating conditions were observed by studying the heat transfer rates for each internal motor component. Table 6 presents the heat transfer rate with respect to the rotating speed at the surface of each major component. While the proportions of heat transferred through convection and conduction at the coil in an ISG operating at $15,000 \mathrm{rpm}$ were $34 \%$ and $66 \%$, respectively, the proportion of heat transferred through convection at a low operating speed of $3000 \mathrm{rpm}$ dropped to $19 \%$. The ratio of heat transferred by convection into the air at the stator, flange, and bracket did not significantly change for all operation ranges. As described above, the ISG, which operates over a wide rotational speed range, may require a heat dissipation design that incorporates heat transfer characteristics that change with the driving speed. A structural improvement design that can improve the conduction heat transfer performance at low speeds must be implemented together with an internal flow passage improvement design that improves the convection heat transfer performance at high speeds. This occurs by reducing the resistance in the inside flow passage and modifying the heat transfer structure by expanding the contact surface between the housing and the motor [20].

Table 6. Heat transfer rates of each surface with respect to rotating speed (@25 $\left.{ }^{\circ} \mathrm{C}\right)$.

\begin{tabular}{lccccccccc}
\hline \multirow{2}{*}{ Heat Transfer Rate $(\mathbf{W})$} & \multicolumn{7}{c}{ Rotating Speed (rpm) } \\
\cline { 2 - 10 } & $\mathbf{3 , 0 0 0}$ & $\mathbf{4 , 5 0 0}$ & $\mathbf{6 , 0 0 0}$ & $\mathbf{7 , 5 0 0}$ & $\mathbf{9 , 0 0 0}$ & $\mathbf{1 0 , 5 0 0}$ & $\mathbf{1 2 , 0 0 0}$ & $\mathbf{1 3 , 5 0 0}$ & $\mathbf{1 5 , 0 0 0}$ \\
\hline Coil $\rightarrow$ Stator & 365.5 & 347.9 & 345.8 & 337.1 & 329.6 & 322.4 & 315.8 & 309.7 & 303.8 \\
\hline Coil $\rightarrow$ Air & 89.6 & 102.8 & 115.8 & 123.3 & 130.7 & 136.3 & 142.5 & 149.5 & 155.8 \\
\hline Stator $\rightarrow$ Flange & 132.8 & 126.4 & 124.1 & 119.8 & 117.1 & 114.2 & 112.1 & 109.5 & 107.1 \\
\hline Stator $\rightarrow$ Bracket & 114.4 & 107.3 & 106.6 & 101.5 & 97.7 & 93.2 & 90.8 & 90.4 & 88.7 \\
\hline Stator $\rightarrow$ Air & 133.8 & 132.2 & 135.8 & 136.9 & 138.1 & 139.5 & 141.0 & 142.6 & 146.0 \\
\hline Bearing $\rightarrow$ Flange & -5.8 & -0.6 & 7.1 & 13.3 & 20.1 & 27.0 & 34.7 & 42.6 & 50.9 \\
\hline Bearing $\rightarrow$ Bracket & 1.5 & 6.29 & 12.6 & 17.9 & 23.9 & 30.0 & 36.7 & 44.3 & 52.0 \\
\hline Flange $\rightarrow$ Air & 116.3 & 119.0 & 123.6 & 126.2 & 130.8 & 135.2 & 141.1 & 146.7 & 152.7 \\
\hline Bracket $\rightarrow$ Air & 101.3 & 102.9 & 109.2 & 109.9 & 112.4 & 114.2 & 118.8 & 125.8 & 132.3 \\
\hline
\end{tabular}

Figure 10 shows the temperatures of the major components with respect to the motor rotation speed. The temperatures of the ISG coil and other major components were highest under the low-speed generation condition of $3000 \mathrm{rpm}$. The coil temperature at $3000 \mathrm{rpm}$ was $104.2{ }^{\circ} \mathrm{C}$, which is a $91.8 \%$ increase compared to the coil temperature at $15,000 \mathrm{rpm}$. When the forced air-cooling method with a centrifugal cooling fan was applied to the ISG, the iron and mechanical losses were reduced at $3000 \mathrm{rpm}$. This in turn reduced the total heat generation by $30 \%$ compared to heat generated at $15,000 \mathrm{rpm}$, as given in Table 4. However, the temperatures of the major components increased as the conduction of the heat from the motor parts to the flange and bracket due to the cooling performance of the centrifugal cooling fan decreased significantly. Kim's [20] analysis of the ISG system also confirms a significant improvement in the heat dissipation performance with the wind volume, which changed with respect to the ISG speed. 


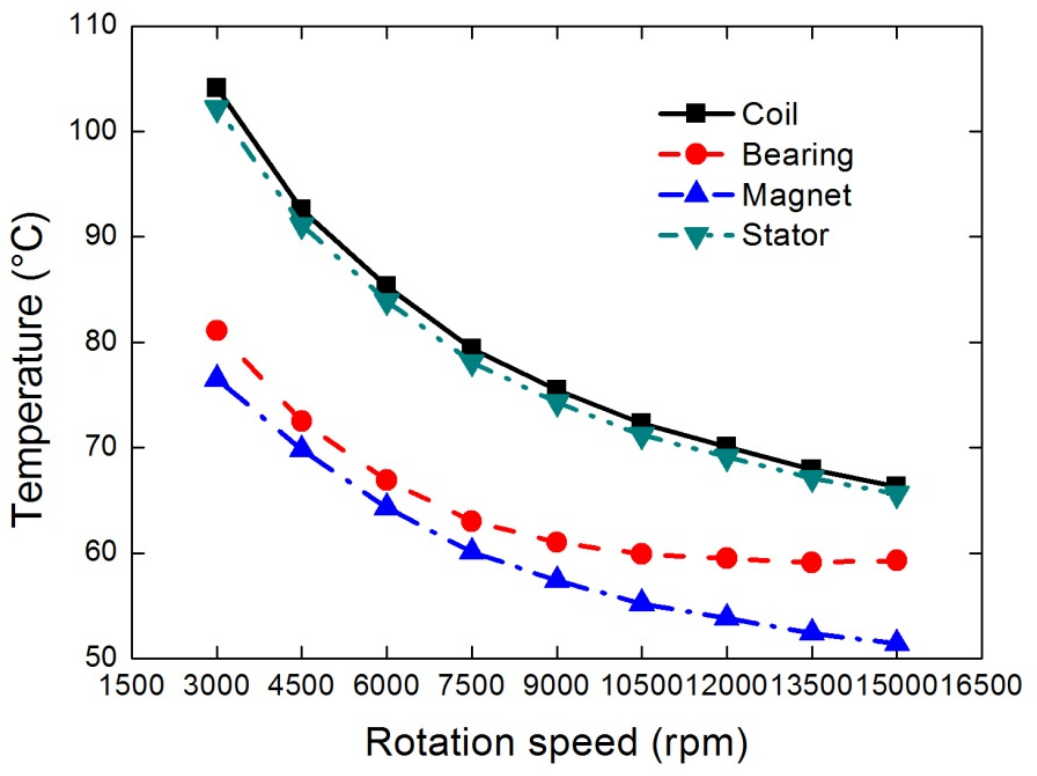

Figure 10. Temperature variations with respect to rotating speed of ISG (ambient temperature: $25{ }^{\circ} \mathrm{C}$ ).

In a vehicle, components can be exposed to various climatic conditions depending on the operation environment. This is especially true for the ISG, which is directly exposed to engine heat owing to its proximity to the engine. Based on such characteristics, a thermal flow analysis was conducted by varying the analysis environment temperature. As shown in Figure 11, the temperatures of the major components in the ISG changed linearly with respect to the ambient temperature. The temperature of each component was estimated to increase by approximately $95{ }^{\circ} \mathrm{C}$ when the ambient temperature increased from $25{ }^{\circ} \mathrm{C}$ to $105^{\circ} \mathrm{C}$ due to increased heat generation in the motor. This is consistent with the result of Kim [20]. The ISG has a durability limit temperature of $200{ }^{\circ} \mathrm{C}$ as it has a coil with an N-type insulator. The coil was heated to $189{ }^{\circ} \mathrm{C}$, so it had a safety temperature margin of approximately $11{ }^{\circ} \mathrm{C}$. The ambient temperature in the engine room of a hybrid electric vehicle is getting decreased as the increase trend of traction motor power.

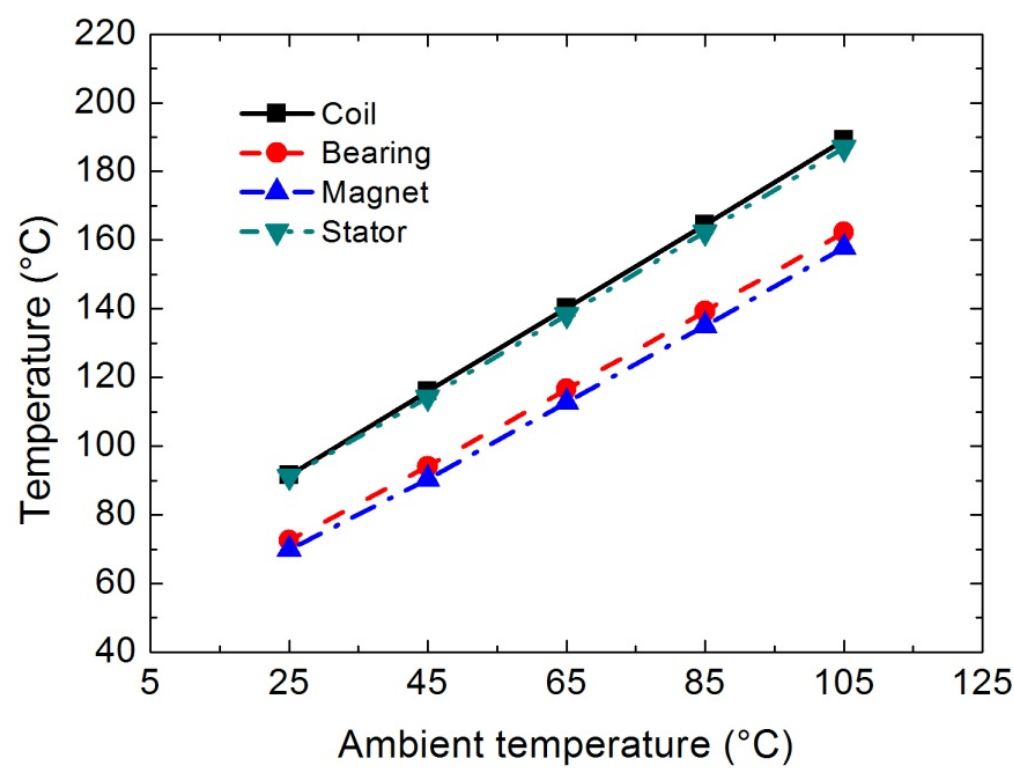

Figure 11. Temperature variations with respect to the ambient temperature of ISG. 


\subsection{Effects of Operating Parameters in Starting Mode}

When operating in the starter mode, the ISG, which must satisfy high torque characteristics, receives a high level of current. As a result, the coil temperature may rapidly increase in a short amount of time. Thus, the operation safety needs to be evaluated. A heat transfer characteristic analysis was conducted for the initial starter stage via a transient analysis of the ISG. Figure 12 shows the changes in the coil and stator temperatures when the ISG was operated as a starting motor at a high ambient temperature of $105^{\circ} \mathrm{C}$.

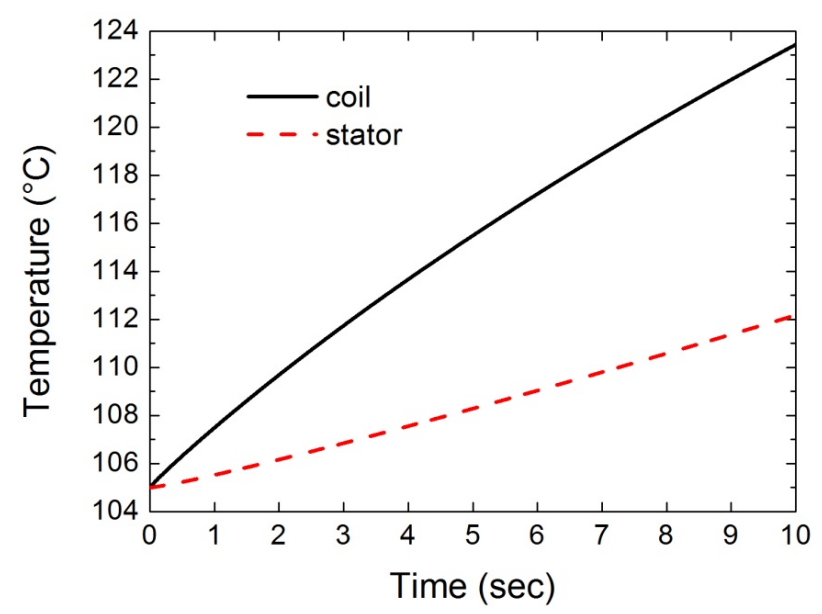

Figure 12. Temperature variations with time for $10 \mathrm{~s}$ at ambient temperature of $105^{\circ} \mathrm{C}$.

The temperature of the ISG coil was estimated to increase by $18{ }^{\circ} \mathrm{C}$ in $10 \mathrm{~s}$ of operation at a high ambient temperature. Conductive heat transfer was dominant over convective heat transfer in the starter stage at the low speed of $800 \mathrm{rpm}$. The resulting significant increase in the temperature at the coil end section can be observed in Figure 13. The temperature deviation between the low and high temperatures of the coil reached up to approximately $24.9^{\circ} \mathrm{C}$, whereas the maximum local temperature increased up to $136.7^{\circ} \mathrm{C}$. For the transient heat flow analysis results in the starting stage, the heat load resulting from the temperature increase was estimated to be modest because the motor is generally operated in this stage over a short period of 1-2 s, despite the high loss of 2-3 kW under the actual initial starting conditions.

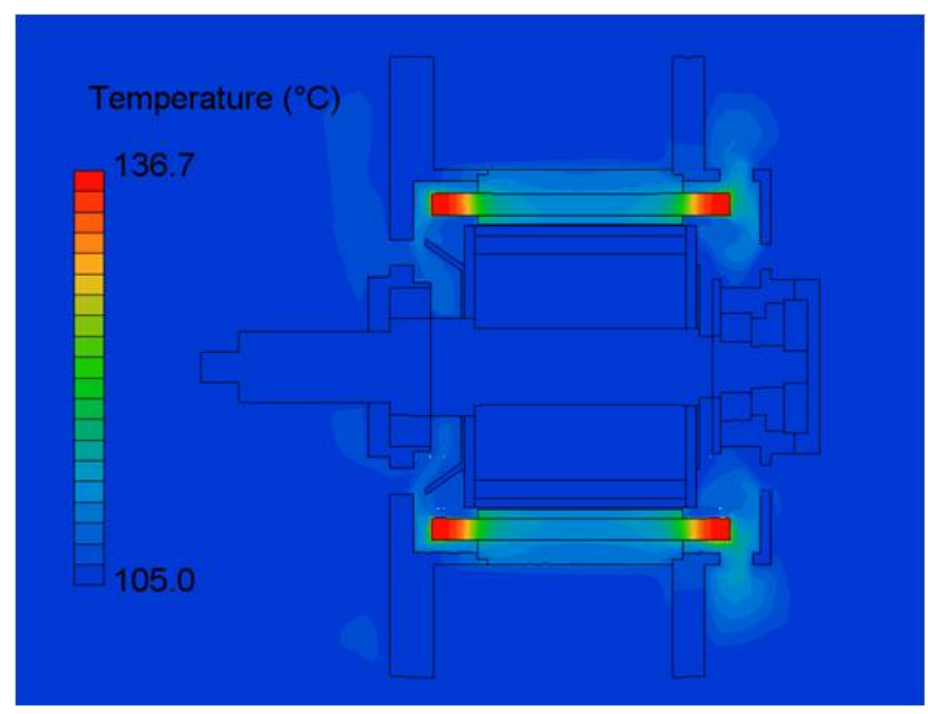

Figure 13. Temperature contours of ISG operating as starter at ambient temperature of $105^{\circ} \mathrm{C}$. 
A Hybrid vehicle with an ISG adopts the idle stop and goes system. Hence, the engine stops when the vehicle is stopped; when the driver intends to go, the vehicle returns to driving mode by starting up the engine. A heat flow analysis was conducted under the conditions where the ISG was restarted and the temperature is saturated because of the generating operation of the ISG. These conditions were taken to reflect the actual driving situations that may arise with these vehicles. A delayed cooling time of $30 \mathrm{~s}$ was used to consider the stopping of the ISG and engine when the vehicle stops. The initial temperature for the heat flow analysis was set to the saturated temperature of the ISG component under generating conditions of $105^{\circ} \mathrm{C}$ and $4500 \mathrm{rpm}$.

The temperature of the coil, which had increased to $189^{\circ} \mathrm{C}$ after the generator operation, decreased by about $6^{\circ} \mathrm{C}$ within $30 \mathrm{~s}$ of stopping. Figure 14 shows that the temperature of the ISG coil increased by approximately $17{ }^{\circ} \mathrm{C}$ to $200{ }^{\circ} \mathrm{C}$ after $10 \mathrm{~s}$ of starting operation by simulation. Similar to the ISG transient heat flow analysis results obtained previously, the highest local temperature was recorded at the coil end. Because it took more than $10 \mathrm{~s}$ to exceed $200^{\circ} \mathrm{C}$, which is the durability limit temperature of the insulator, it was concluded that there would be no thermal problems considering the short operation time during actual driving.

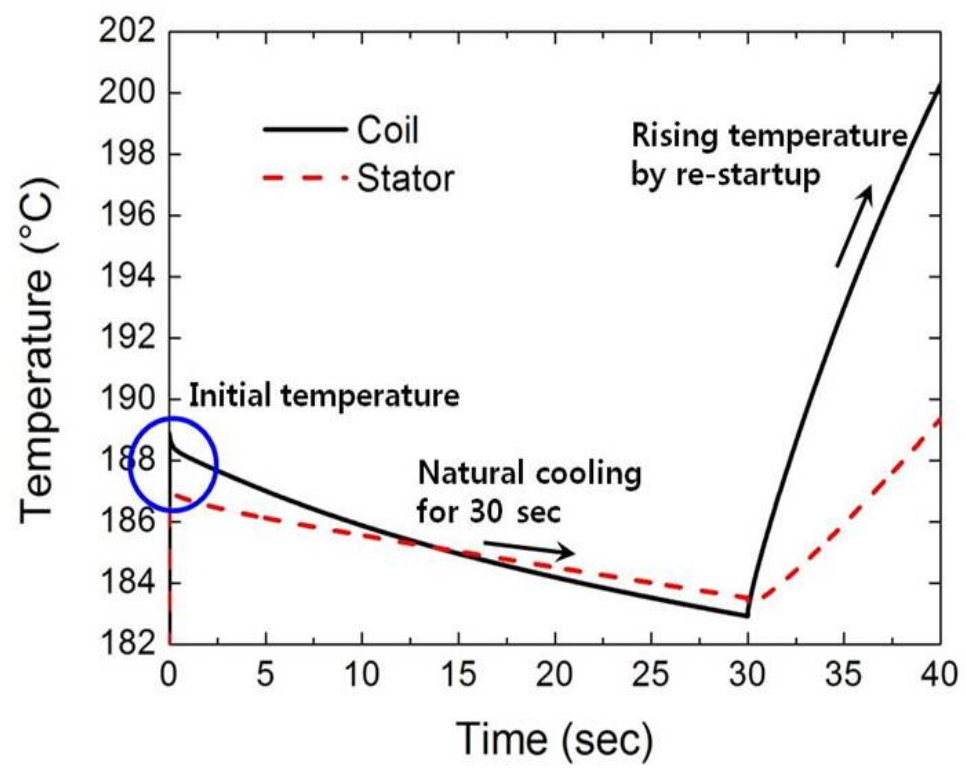

Figure 14. Temperature variation after startup for $10 \mathrm{~s}$ after natural cooling for $30 \mathrm{~s}$ by simulation.

\subsection{Experimental Result and Analysis Validation}

As shown in Figure 15, to reflect the heat loss attributed to the ISG testing environment, heat flow analysis was conducted by considering the shape model installed on the jig. As shown in Figure 16, the temperatures of the coil and stator core measured in the motor dynamometer experiment increased to $52.2{ }^{\circ} \mathrm{C}$. The difference between this temperature and the temperature from the heat flow analysis results, which were used for analysis validation and which recorded the maximum temperature, was approximately $1.8{ }^{\circ} \mathrm{C}$ and $2.4{ }^{\circ} \mathrm{C}$ for the coil and stator core, respectively. In terms of the increase in temperature, these differences showed approximate errors of $6.6 \%$ and $8.7 \%$, respectively. These errors could be attributed to the fact that the heat flow analysis model could not simulate all of the physical phenomena generated in the performance experiment using an actual prototype. 


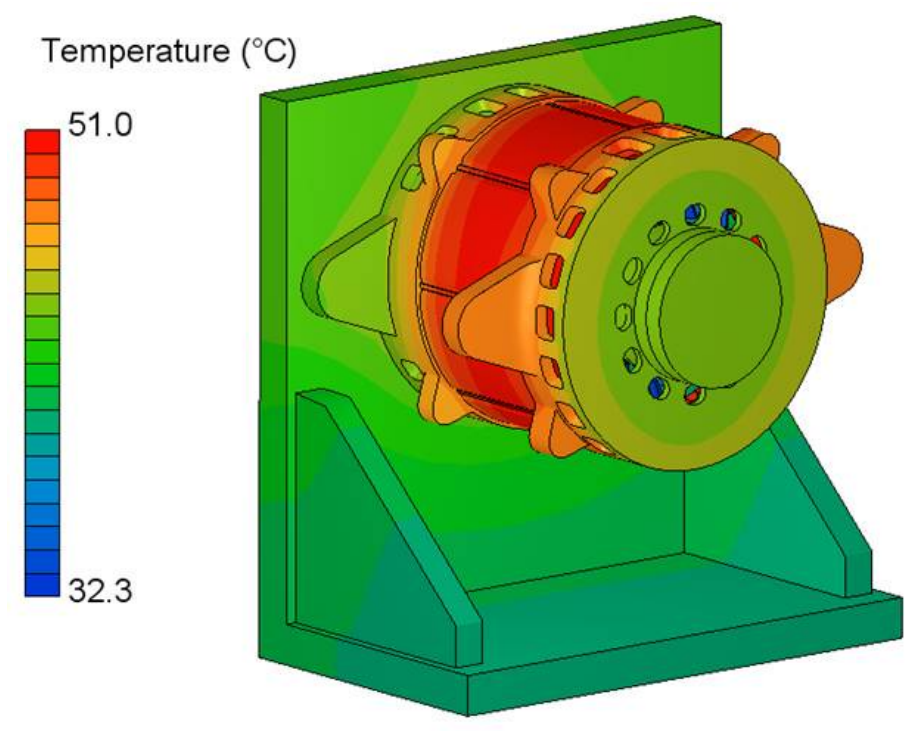

Figure 15. Temperature contours of ISG for reliability verification.

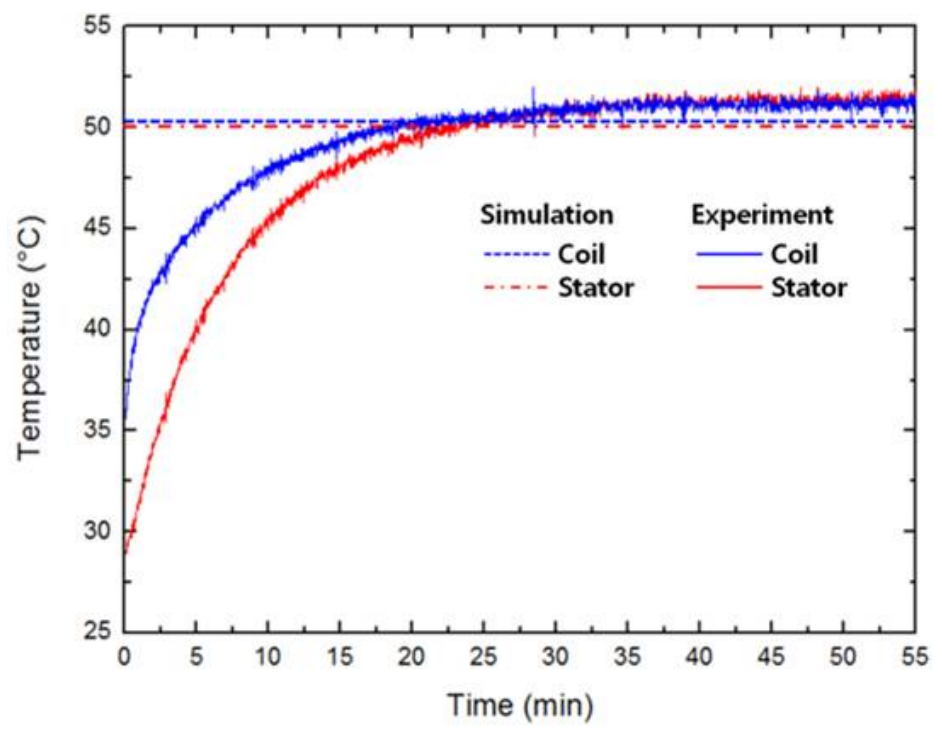

Figure 16. Comparison of analysis and experimental results.

\section{Conclusions}

The present study confirmed the effects of the operating parameters on the thermal performance of an integrated starter generator motor in a hybrid electric vehicle. The effects were studied by analyzing the changes in the heat dissipation characteristics with respect to the ambient temperature and rotation speed of a 42 V ISG operating as a generator in a hybrid vehicle. Operation safety was confirmed through a transient state heat flow analysis of the temperature variation and heat dissipation characteristics of the starting period, during which significant losses are generated over a short period of time.

The thermal performance of the forced air-cooled ISG varied significantly with respect to the rotating speed. The component temperatures increased significantly under an operating condition of $3000 \mathrm{rpm}$, even though the heat value had decreased by $30 \%$ compared to the heat value under the operating condition of $15,000 \mathrm{rpm}$. In the coil, the temperature increased by $92 \%$ to $104{ }^{\circ} \mathrm{C}$. The heat dissipation characteristics, which vary with respect to the rotation speed, were also observed through the variation 
in the heat transfer rate per component. The heat transfer rate for cooling by convection in the coil increased from $19 \%$ at $3000 \mathrm{rpm}$ to $34 \%$ at $15,000 \mathrm{rpm}$. A cooling design that considers the changes in the thermal characteristics with respect to the operating speed of the air-cooled ISG is required.

The heat flow analysis was conducted considering the harsh environment of the engine room where the ISG operates. The coil temperature of an ISG operated at $4500 \mathrm{rpm}$ and $105^{\circ} \mathrm{C}$ was found to increase up to $189^{\circ} \mathrm{C}$. However, considering the durability limit of the N-type coil insulator applied to the coil, this increase is not a problem.

The transient state heat flow analysis was conducted for a situation where the motor was started after $30 \mathrm{~s}$ in the stopped state to consider the harsh conditions of an ISG operating as a starter in actual vehicles. After the coil had cooled by approximately $6{ }^{\circ} \mathrm{C}$ owing to natural convection when the motor was in the stopped state, the temperature increased to $200^{\circ} \mathrm{C}$ after an increase of $17^{\circ} \mathrm{C}$ because $10 \mathrm{~s}$ had elapsed since the starting operation. Although concentrated temperature increases were confirmed in the coil end because of the decreased thermal effects of the centrifugal cooling fan owing to the low rotation velocity of $800 \mathrm{rpm}$, this should not be a problem considering the short operating time.

\section{Acknowledgments}

This study was carried out as a part of the Industrial Source Technology Development project sponsored by the Ministry of Knowledge Economy. This work was supported by the 2015 Yeungnam University Research Grant. The support from Komotek Co., Ltd. is also greatly appreciated.

\section{Author Contributions}

Sung Chul Kim and Moo-Yeon Lee organized the overall numerical analysis and experimental verification. Dong Hyun Lim commented on the results and conclusions. All the authors reviewed the manuscript.

\section{Conflicts of Interest}

The authors declare no conflict of interest.

\section{Nomenclature}

$B \quad$ peak magnetic flux density $(\mathrm{T})$

$f \quad$ frequency $(\mathrm{Hz})$

Ieff effective phase current (Arms)

$i_{\max } \quad$ maximum current (A)

$k_{a} \quad$ anomalous eddy current loss coefficient

$k_{e} \quad$ eddy current coefficient

$k_{h} \quad$ hysteresis coefficient

$L_{s p} \quad$ average turn length (m)

$m_{\text {rot }} \quad$ rotor part mass $(\mathrm{kg})$

$N \quad$ rotational speed (rpm)

$n_{r} \quad$ number of bearing 


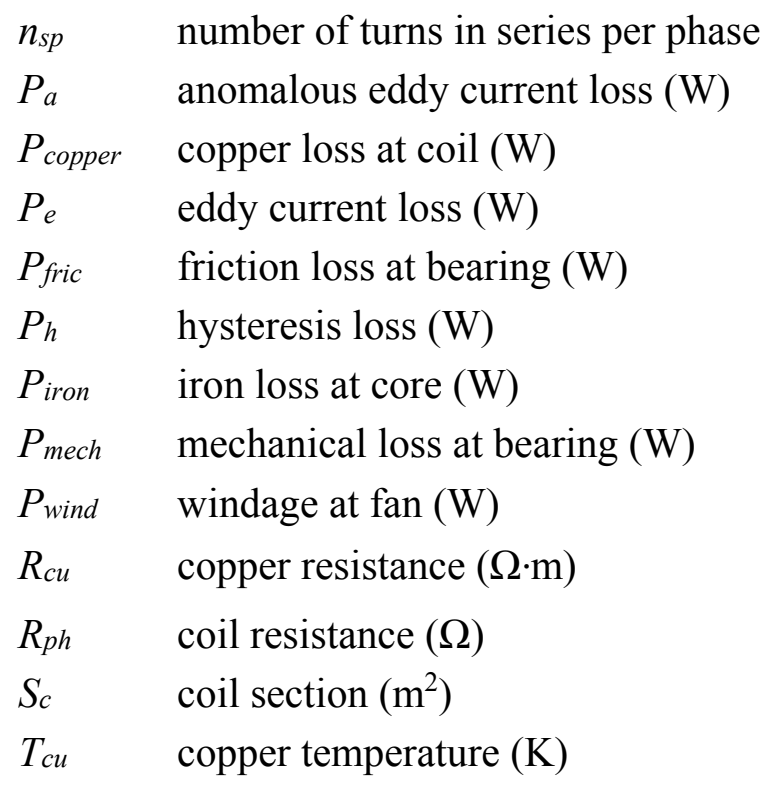

\section{References}

1. Anandakumaran Nair, K.R.; Narayanan, V. $42 \mathrm{~V}$ System for Future Passenger Cars; SAE Technical Paper, No. 2001-28-0019; SAE International: Warrendale, PA, USA, 2001.

2. Lukic, S.M.; Emadi, A. Effects Electrical Loads on 42V Automotive Power Systems; SAE Technical Paper, No. 2003-01-2257; SAE International: Warrendale, PA, USA, 2003.

3. Bitsche, O.; Gutmann, G. Systems for hybrid cars. J. Power Sources 2004, 127, 8-15.

4. Viorel, I.A.; Szabo, L.; Lowenatein, L.; Stet, C. Integrated starter-generators for automotive applications. Acta Electrotehnica 2004, 46, 255-260.

5. Simopoulos, G.N.; MacBain, J.A.; Schneider, E.D.; Wingeier, E.W. Fuel Economy Improvements in an SUV Equipped with an Integrated Starter Generator. In Proceedings of the SAE International Truck \& Bus Meeting \& Exhibition, Chicago, IL, USA, 12-14 November 2001.

6. Rosero, J.A.; Romeral, L.; Ortega, J.A.; Rosero, E. Short-circuit detection by means of empirical mode decomposition and Wigner-Ville distribution for PMSM running under dynamic condition. IEEE Trans. Ind. Electron. 2009, 56, 4534-4547.

7. Qiao, M.; Zhang, X.; Ren, X. Research of the mathematical model and sudden symmetrical short circuit of the multi-phase permanent-magnet motor. Int. Conf. Power Syst. Technol. 2002, 2, 769-773.

8. Kim, K.C.; Lim, S.B.; Koo, D.H.; Lee, J. The shape design of permanent magnet for permanent magnet synchronous motor considering partial demagnetization. IEEE Trans. Magn. 2006, 42, 3485-3487.

9. Kim, K.H.; Choi, D.U.; Gu, B.G.; Jung, I.S. Fault model and performance evaluation of an inverter-fed permanent synchronous motor under winding shorted turn and inverter switch open. IET Electr. Power Appl. 2010, 4, 214-225.

10. Kimotho, J.; Hwang, P. Thermal Management of Electric Vehicle BLDC Motor; SAE Technical Paper, No. 2011-28-0134; SAE International: Warrendale, PA, USA, 2011.

11. Lim, D.H.; Kim, S.C. Thermal performance of oil spray cooling system for in-wheel motor in electric vehicles. Appl. Therm. Eng. 2014, 63, 577-587. 
12. Kim, K.S.; Lee, B.H.; Jung, J.W.; Hong, J.P. Thermal analysis of water cooled ISG based on a thermal equivalent circuit network. J. Electr. Eng. Technol. 2014, 9, 742-747.

13. MotorPro. User's Guide Solver Reference; Version 2.6.B; Komotek: Sungnam, Korea, 2004.

14. Kim, D.G.; Kim, S.C. An analysis study for thermal design of ISG (Integrated Starter Generator) for hybrid electric vehicle. Trans. Korean Soc. Automot. Eng. 2013, 21, 120-127.

15. SC/Tetra. User's Guide Solver Reference; Version 7; Software Cradle Co., Ltd.: Osaka, Japan, 2007.

16. Kato, M.; Launder, B.E. The Modeling of Turbulent Flow around Stagnation and Vibrating Square Cylinders. In Proceedings of the 9th Symposium on Turbulent Shear Flow, Kyoto, Japan, 16-18 August 1993; pp. 10-14.

17. Fakhfakh, M.A.; Kasem, M.H.; Tounsi, S.; Neji, R. Thermal analysis of a permanent magnet synchronous motor for electric vehicles. J. Asian Electr. Veh. 2008, 6, 1145-1151.

18. Jang, S.M.; Cho, H.W.; Choi, S.K. Design and analysis of a high-speed brushless DC motor for centrifugal compressor. IEEE Trans. Magn. 2007, 43, 2573-2575.

19. Trippett, R.J. A high-speed rolling-element bearing loss investigation. J. Eng. Gas Turbines Power 1978, 100, 40-45.

20. Kim, S.C. Thermal performance of motor and inverter in an integrated starter generator system for a hybrid electric vehicle. Energies 2013, 6, 6102-6119.

(C) 2015 by the authors; licensee MDPI, Basel, Switzerland. This article is an open access article distributed under the terms and conditions of the Creative Commons Attribution license (http://creativecommons.org/licenses/by/4.0/). 